\title{
Analysis of three epoetin alpha products by $L C$ and $L C-M S$ indicates differences in glycosylation critical quality attributes, including sialic acid content
}

\section{Article}

Published Version

\section{Open Access}

Thomson, R. I., Gardner, R. A., Strohfeldt, K., Fernandes, D. L., Stafford, G. P., Spencer, D. I. R. and Osborn, H. M. I. (2017) Analysis of three epoetin alpha products by LC and LCMS indicates differences in glycosylation critical quality attributes, including sialic acid content. Analytical Chemistry, 89 (12). pp. 6455-6462. ISSN 0003-2700 doi:

https://doi.org/10.1021/acs.analchem.7b00353 Available at https://centaur.reading.ac.uk/70676/

It is advisable to refer to the publisher's version if you intend to cite from the work. See Guidance on citing.

To link to this article DOI: http://dx.doi.org/10.1021/acs.analchem.7b00353

Publisher: American Chemical Society

All outputs in CentAUR are protected by Intellectual Property Rights law, including copyright law. Copyright and IPR is retained by the creators or other copyright holders. Terms and conditions for use of this material are defined in the End User Agreement. 


\section{www.reading.ac.uk/centaur}

\section{CentAUR}

Central Archive at the University of Reading

Reading's research outputs online 


\section{Analysis of Three Epoetin Alpha Products by LC and LC-MS Indicates Differences in Glycosylation Critical Quality Attributes, Including Sialic Acid Content}

Rebecca I. Thomson, ${ }^{\dagger}$ Richard A. Gardner, ${ }^{\dagger}$ Katja Strohfeldt, ${ }^{\dagger}$ Daryl L. Fernandes, ${ }^{\ddagger}$ Graham P. Stafford, ${ }^{\S}$ Daniel I. R. Spencer, $*$, and Helen M. I. Osborn* $* \dagger$

${ }^{\dagger}$ Reading School of Pharmacy, University of Reading, Reading, Berkshire RG6 6AP, United Kingdom

${ }^{\ddagger}$ Ludger, Ltd., Culham Science Centre, Abingdon, Oxfordshire OX14 3EB, United Kingdom

${ }^{\S}$ Integrated BioSciences, School of Clinical Dentistry, University of Sheffield, Sheffield, S10 2TA, United Kingdom

\section{Supporting Information}

ABSTRACT: Erythropoietin (EPO) is one of the main therapeutics used to treat anemic patients, greatly improving their quality of life. In this study, biosimilars Binocrit and a development product, called here CIGB-EPO, were compared to the originator product, Eprex. All three are epoetin alpha products, reputed to have similar glycosylation profiles. The quality, safety, and efficacy of this biotherapeutic depend on the following glycosylation critical quality attributes (GCQAs): sialylation, N-glycolyl-neuraminic acid (Neu5Gc) content, branching, $N$-acetyl-lactosamine (LacNAc) extensions, and $O$-acetylation pattern. Reverse-phase ultra-high-pressure liquid

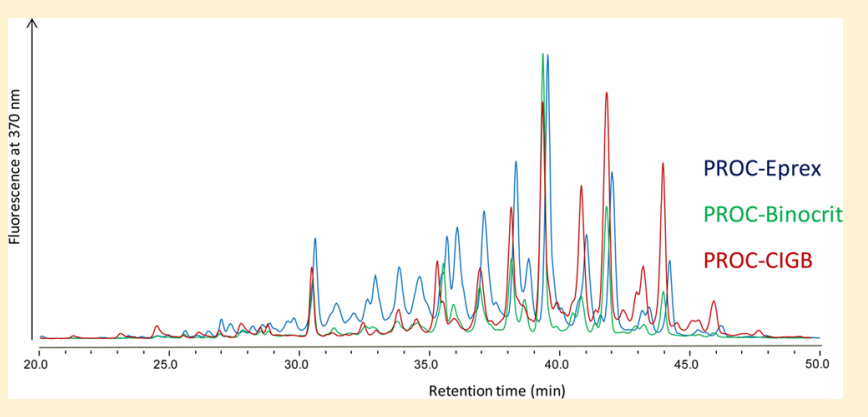
chromatography (RP-UHPLC) analysis of acid-released, 1,2-diamino-4,5-methylenedioxybenzene (DMB) labeled sialic acid derivatives and hydrophilic interaction liquid chromatography (HILIC) in combination with mass spectrometry (HILICUHPLC-MS) of procainamide (PROC) labeled N-glycans were the analytical tools used. An automated method for enzymatic release and PROC labeling was applied for the first time to the erythropoiesis stimulating agent (ESA) products, which facilitated novel, in-depth characterization, and allowed identification of precise structural features including the location of $O$-acetyl groups on sialic acid (SA) moieties. Samples were digested by a sialate- $O$-acetylesterase $(\mathrm{NanS})$ to confirm the presence of $O$-acetyl groups. It was found that Eprex contained the greatest relative abundance of $O$-acetylated derivatives, Binocrit expressed the least Neu5Gc, and CIGB-EPO showed the greatest variety of high-mannose-phosphate structures. The sialylation and LacNAc extension patterns of the three ESAs were similar, with a maximum of four $N$-acetyl-neuraminic acid (Neu5Ac) moieties detected per glycan. Such differences in SA derivatization, particularly $O$-acetylation, could have consequences for the quality and safety of a biotherapeutic, as well as its efficacy.

E rythropoietin (EPO) is a mammalian glycoprotein hormone, mainly produced in the kidney, that regulates the level of red blood cells in circulation. ${ }^{1}$ It is recognized as a medicinal product for the treatment of anemia due to renal failure or chemotherapy. ${ }^{2}$ Unfortunately, EPO is often also exploited as a doping strategy by athletes. ${ }^{3}$ Detection of the illegal use of synthetic EPO analogues, known as erythropoiesis stimulating agents (ESAs), is complicated by the ongoing development of biotherapeutics with longer bloodstream lifetimes. ${ }^{4}$

The first commercially available ESA was an epoetin alpha brand-named Eprex. ${ }^{2}$ Expiration of the epoetin alpha patent in Europe in 2004 allowed "biosimilar" ESAs to enter the market. When developing a biosimilar it must be proven that the proposed biotherapeutic yields neither a better nor worse result compared to the originator product. ${ }^{5}$ Moreover, structural information must be presented to the relevant regulating body. For EPO, which contains highly sialylated glycans, product glycosylation may differ between biosimilars, and this should therefore be monitored by defining and measuring glycoprotein critical quality attributes (GCQAs).

Particularly important components of the glycan chain are sialic acids (SAs). Of particular note, an ESA containing up to eight extra SA residues shows a 3-fold increase in serum halflife. $^{6}$ Moreover, the main difference between human serum EPO and recombinant human EPO is a lack of tetra-sialylated structures in the former. ${ }^{7}$

$\mathrm{N}$-Linked rather than $\mathrm{O}$-linked glycans influence the efficacy of EPO, with the SA residues on the $N$-glycans being the key components necessary for in vivo activity. ${ }^{8,9}$ Within the $N$ glycan characteristics of EPO, the length of lactosamine (LacNAc) repeats and amount of structural branching can

Received: January 27, 2017

Accepted: May 16, 2017

Published: May 16, 2017 
also affect the lifetime of a biosimilar. ${ }^{10}$ O-Acetylation of SAs, which has been shown to increase hydrophobicity of this sugar and inhibit sialidase action, ${ }^{11}$ has been hypothesized as another mechanism to retain glycoproteins in the bloodstream. ${ }^{12}$ Aside from $\mathrm{N}$-acetylneuraminic acid (Neu5Ac) content, the presence of $\mathrm{N}$-glycolylneuraminic acid (Neu5Gc) must be monitored, as humans express antibodies directed against it that cause rapid clearance of Neu5Gc-containing biotherapeutics from the bloodstream. ${ }^{13}$

As the originator product, Eprex is taken as the standard ESA against which all other treatments are compared. ${ }^{14}$ The first approved biosimilar to Eprex, also an epoetin alpha, was brandnamed "Binocrit". CIGB-EPO is an epoetin alpha still under development. ${ }^{15}$ Eprex and Binocrit have both been shown to consist of six isoforms, but with differing abundances. ${ }^{16}$

As all three ESAs fall under the category of epoetin alpha, denoting Chinese hamster ovary $(\mathrm{CHO})$ cell line manufacture, ${ }^{17}$ their glycosylation profiles are expected to be very similar although not identical, as the biochemical processes depend on a range of conditions that can vary between manufacturers and even between batches. ${ }^{5}$ The monitoring of such variations in glycan structure is essential as it could potentially account for differences in immunogenicity, half-life of the ESAs, and efficacy of the products. ${ }^{2,18}$

In view of the impact of SA profile on the lifetime of ESAs in the bloodstream and its further possible role in adverse effects, the present investigation centered on this aspect, particularly the lesser examined $O$-acetylation pattern. The branched nature of $\mathrm{N}$-glycans, coupled with the different ring sizes and anomericity of their constituent monosaccharides, make them notoriously difficult to analyze. ${ }^{19}$ Thus, the approach taken was to use liquid chromatography coupled with mass spectrometry (LC-MS) employing two different fluorescent tags: DMB for identification of different $O$-acetylated SA derivatives and PROC labeling of released $\mathrm{N}$-glycans. O-Acetylation content was confirmed using an $\mathrm{O}$-acetylesterase. A Hamilton Microlab STARlet liquid-handling robot was used with a view to automating sample preparation and facilitating faster analysis of glycans labeled with an LC-MS compatible fluorophore tag.

\section{MATERIALS AND METHODS}

Erythropoiesis Stimulating Agents (ESAs). Licensed EPO products Eprex (Janssen-Cilag) and Binocrit (Sandoz) were purchased from a certified pharmacy. A development ESA, named CIGB-EPO, was a gift from Antonio Vallin, Center for Molecular Immunology, Cuba. The samples were buffer exchanged into water using Vivaspin 6 devices (MWCO 10,000) (Sartorius). To aid maximum possible recovery of the sample, the Vivaspins were rinsed twice with water and the washes added to the previously exchanged material. Each ESA was buffer-exchanged in the same way to enable direct comparison of results. The samples were then stored below 0 ${ }^{\circ} \mathrm{C}$. Method outlines are given in the following subsections and more detail can be found in the Supporting Information.

Sialic Acid (SA) Analysis. Release and fluorescence labeling of the SAs was carried out using a DMB sialic acid release and labeling kit (Ludger) as previously described in the literature. $^{20}$ The released and labeled samples, standard, and reference thus obtained were diluted by 1 in 10 and analyzed by RP-HPLC on a LudgerSep-uR2 C18 $100 \times 2.1 \mathrm{~mm}$, i.d. $1.7 \mu \mathrm{m}$ column, elution gradient methanol/acetonitrile/water. An Ultimate 3000 UHPLC (Thermo) equipped with a fluorescence detector (FD) was used (excitation wavelength $373 \mathrm{~nm}$, emission wavelength $448 \mathrm{~nm}$ ) and data were analyzed using the Chromeleon 7.2 software. Each ESA was run three times, that is three separately released and labeled samples.

$\mathrm{N}$-Glycan Analysis. Denaturation, Release, and Labeling. A Hamilton Microlab STARlet liquid-handling robot was used for sample preparation of the $\mathrm{N}$-glycans in a similar manner to that previously published. ${ }^{21,22} \mathrm{~A}$ procainamide labeling kit (Ludger) containing 2-picoline borane was used, as described by Kozak et al., again employing the Hamilton Microlab STARlet liquid-handling robot. ${ }^{23}$ Samples were analyzed by HILIC-LC on an Ultimate 3000 UHPLC using a BEH-Glycan $1.7 \mu \mathrm{m}, 2.1 \times 150 \mathrm{~mm}$ column (Waters) at $40{ }^{\circ} \mathrm{C}$ with a fluorescence detector $\left(\lambda_{\mathrm{ex}}=310 \mathrm{~nm}, \lambda_{\mathrm{em}}=370 \mathrm{~nm}\right)$, controlled by Bruker HyStar 3.2. Buffer A was $50 \mathrm{mM}$ ammonium formate made from LudgerSep N Buffer stock solution, pH4.4 [LS-NBUFFX40]; buffer B was acetonitrile (acetonitrile 190 far UV/ gradient quality; Romil \#H049). Samples were injected in $24 \%$ aqueous $/ 76 \%$ acetonitrile; injection volume $25 \mu \mathrm{L}$. Chromeleon data software version 7.2 with a cubic spline fit was used to allocate glucose unit (GU) values to peaks. PROC labeled glucose homopolymer was used as a system suitability standard as well as an external calibration standard for GU allocation for the system. ${ }^{24}$ Each ESA was run in quadruplicate.

Mass Spectrometry (MS). A Bruker amaZon Speed ETD electrospray mass spectrometer was coupled directly after the UHPLC FD without splitting. The instrument scanned samples in maximum resolution mode, positive ion setting, MS scan + three MS/MS scans, nebulizer pressure 14.5 psi, nitrogen flow $10 \mathrm{~L} / \mathrm{min}$, capillary voltage $4500 \mathrm{~V}$. MS/MS was performed on three ions in each scan sweep with a mixing time of $40 \mathrm{~ms}$. Mass spectrometry data were analyzed using the Bruker Compass DataAnalysis 4.1 software in combination with Bruker Proteinscape 4.0 for assisting peak assignments to a Ludger EPO glycan database. Structures were identified by comparing LC, MS, and MS/MS data.

O-Acetylesterase Digest. Sets of PROC-labeled N-glycans released from each ESA were subjected to enzyme digest by NanS (LZ-ACASE-KIT, Ludger), specific for the removal of 7-, 8 -, and 9-O-acetyl groups, as described in the literature. ${ }^{25,26}$ Briefly, $1 \mu \mathrm{L}$ of enzyme $(0.7 \mathrm{mg} / \mathrm{mL})$ was added to each sample and buffer ( $50 \mathrm{mM}$ sodium acetate buffer, $\mathrm{pH} 5.5)$ was added to give a total volume of $10 \mu \mathrm{L}$ before incubation at $37^{\circ}$ for $16 \mathrm{~h}$. The enzyme was removed using a protein-binding plate and the resulting samples analyzed by HILIC-UHPLC. Each digest was only carried out once.

\section{RESULTS}

1,2-Diamino-4,5-methylenedioxybenzene (DMB) Analysis. DMB-labeling of acid released SAs is an established method allowing comparison between samples and concurrently run references and standards. The sialic acid reference panel (SRP), derived from a mild digest of bovine submaxillary mucin, is well-known and characterized in the literature. ${ }^{27}$ SRP and GPEP (a biantennary disialylated $N$-glycan linked to peptide KVANKT) acted as positive controls to confirm successful labeling. Comparison of GPEP with the SRP showed that it contained just NeuSAc as expected. Excluding the free dye peak at retention time $5.10 \mathrm{~min}$, the relative abundance of each SA structure present in the ESA samples was calculated (Figure 1). The results showed good repeatability (Figure S1). Where the average relative area of the peak is $>0.5 \%$, the relative standard deviation (RSD) value should be $<10 \%$, but where the average relative area of the peak is $<0.5 \%$, the RSD 


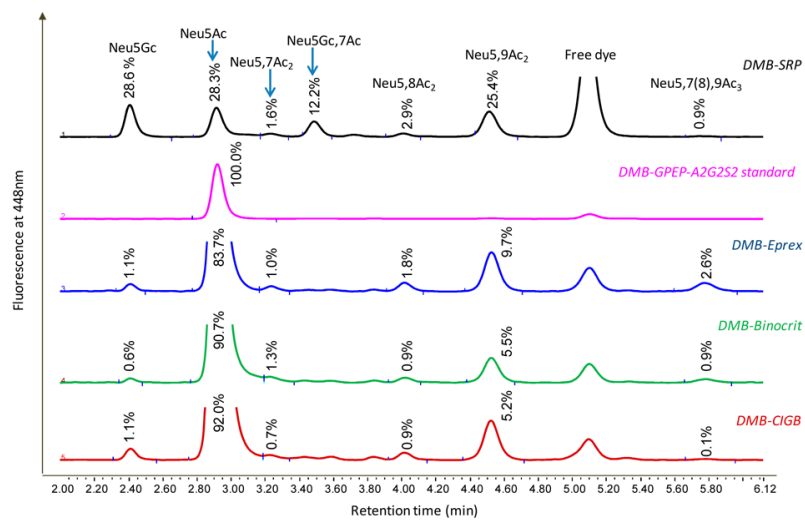

Figure 1. RP-HPLC traces of standard and ESA DMB derivatives. Relative percentage area is given above each peak. Mono- and di-Oacetylated SAs contained in samples can be identified by comparison to the SRP. Assignments based on Klein et al. ${ }^{27}$

value should be $<30 \%$, as indicated in green (Figures S2-S4 and Tables S1-S3). This was the case for most of the peaks in the current study. Absolute abundances have not been calculated because the quantity of material that was buffer exchanged can only be estimated.

Although the $O$-acetylation pattern of ESA products is clearly similar in terms of the types of SA structure present, there are differences in the relative abundances of many species. Strikingly, Neu5Gc is expressed to a similar degree in both Eprex and CIGB-EPO, whereas Binocrit shows almost half the relative quantity. On the other hand, similarity between Binocrit and CIGB-EPO can be seen in the relative abundance of N-8-O-acetyl-acetylneuraminic acid $\left(\mathrm{Neu} 5,8 \mathrm{Ac}_{2}\right)$ and $\mathrm{N}-9-\mathrm{O}-$ acetyl-acetylneuraminic acid $\left(\mathrm{Neu} 5,9 \mathrm{Ac}_{2}\right)$, but the relative abundance of these two species in Eprex is double. Di-Oacetylation in the form of Neu5,7,9 $\mathrm{Ac}_{3}$ is present in all three ESA products, but in a broad range of $0.1-2.6 \%$.

Procainamide (PROC) Analysis. UHPLC traces for the PNGase F released, PROC-labeled N-glycans of the ESAs were obtained (Figure 2). Samples were run in quadruplicate with good repeatability evidenced by low RSD values (Figures S11S13 and Tables S4-S6). Integration of peaks was achieved using Chromeleon software on an Ultimate 3000 UHPLC. Glycan peak (GP) numbers were assigned by taking into account GU values alongside carbohydrate content of peaks. The structures contained in each LC peak were identified by examining the corresponding MS and MS/MS data (Tables S7-S9) and the traces have been labeled accordingly. The "Oxford notation" nomenclature has been used for the linear notation but without strict adherence to linkage type. ${ }^{28}$

In general, the more highly $\mathrm{O}$-acetylated a structure, the sooner it elutes because of increased hydrophobicity, and the more branching or chains that the glycans form, the longer the retention time. Some structures, such as FA2G2S2(Ac)2, appear in all ESAs, albeit at slightly different GU values (GP 3, 6, and 7 of Figure 2). Shifts in GU between structures of apparently the same constituents are usually due to the presence of different isomers. The majority of structures are believed to be core fucosylated, as previous studies have found this to be the most likely position of the fucose residue. ${ }^{29}$

Primarily, glycan structures were assigned according to the MS data. The MS/MS data obtained was used to determine the position of $O$-acetyl groups on SA residues. Of particular relevance to this study were the fragments: $\mathrm{m} / \mathrm{z} 657.16$
(HexHexNAcNeuAc), $m / z$ 699.17 (HexHexNAcNeuAc$(+1 \mathrm{Ac})$ ), and $m / z 741.18$ (HexHexNAcNeuAc$(+2 \mathrm{Ac}))$. If found, these fragments provide evidence as to where the $O$ acetyl groups were located. For example, the Eprex MS/MS of peak $\mathrm{m} / z 891.79$ fragmented into $\mathrm{m} / z 657$ and $\mathrm{m} / \mathrm{z} 741$ alongside an absence of $\mathrm{m} / z$ 699, indicating that the two $O$ acetyl groups were located on the same SA (Figure 3 and Tables S10 and S11). Conversely, $\mathrm{m} / z$ 877.61 contained fragments $\mathrm{m} / z 657$ and $\mathrm{m} / z 699$ without $\mathrm{m} / \mathrm{z} 741$, showing that a SA was singly $O$-acetylated. Also of interest in this study was $\mathrm{m} / \mathrm{z} 672.23$, corresponding to Neu5Gc (Figure S14). Limitations of the MS method used for peak identification include the inability to distinguish between greater branching of the core structure of the glycan as opposed to extended LacNAc repeats, such as between FA4G4S3 and FA3G3S3(LacNAc)1 (GP 33 of Figure 2). Strengths of the method include the high fluorescence intensity of derivatives and good sensitivity. Use of the milder ESI as opposed to matrix assisted laser desorption/ionization (MALDI) ensured that there was neither loss of SA moieties nor migration of $\mathrm{O}$-acetyl groups. ${ }^{12,19}$

Enzyme Digest. PROC-labeled ESAs were analyzed by UHPLC before and after digestion with an $O$-acetylesterase that removed all 7-, 8-, and 9-O-acetyl moieties of SA and the resulting traces were compared (Figure 4). As expected, peaks from the original sample that contained such $O$-acetylated SA derivatives disappeared after incubation with the enzyme. This was particularly pronounced for Eprex, which shows a markedly altered profile after digest, thus confirming the highly $\mathrm{O}$ acetylated nature of its $\mathrm{N}$-linked glycans.

\section{DISCUSSION}

When developing a glycoprotein as a biotherapeutic, quality, safety, and efficacy are the most important criteria to consider. ${ }^{18}$ For an ESA, the GCQAs affecting these criteria are sialylation, level of Neu5Gc, core glycan structures, LacNAc extensions, and arguably, acetylation pattern. ${ }^{30}$

Sialylation is readily present in all of the ESAs analyzed, with a maximum of four SA moieties present on some glycan structures, similar to literature reports. ${ }^{7,30}$ A greater number of SA moieties is known to extend the half-life of an erythropoietin, thus improving its efficacy. ${ }^{6}$ A greater degree of $\mathrm{O}$-acetylation of $\mathrm{SA}$ is also thought to improve therapeutic half-life as its presence has been shown to slow the action of sialidases. ${ }^{11,12}$ In the literature, although still debated, there is evidence to suggest that migration from the 7- to the 9-position of the SA structure is not purely because of $\mathrm{pH}$ changes but is accelerated by a so-called "migrase". ${ }^{31}$ Once at the 9-position, the $O$-acetyl group's removal can be effected by the sialidase, leaving the underlying galactose residue exposed to hepatocyte receptors. ${ }^{32}$ Arguably, therefore, not only the extent but also the position of an $\mathrm{O}$-acetyl group could affect the half-life of a biotherapeutic.

Although DMB has previously been used to label SAs released from ESAs, the authors of that study did not take advantage of the DMB method in order to calculate the relative abundances of all derivatives detected, nor was the process automated..$^{30}$ In the present study, DMB analysis shows the presence of mono- and di-O-acetylation of NeuSAc in all three ESA products, but in varying relative abundances. Eprex comprises a relative abundance of $15.1 \%$ of $O$-acetylated SA, compared to $8.6 \%$ for Binocrit and $6.9 \%$ for CIGB-EPO (Figure 1). This observation is supported by the PROC labeled 

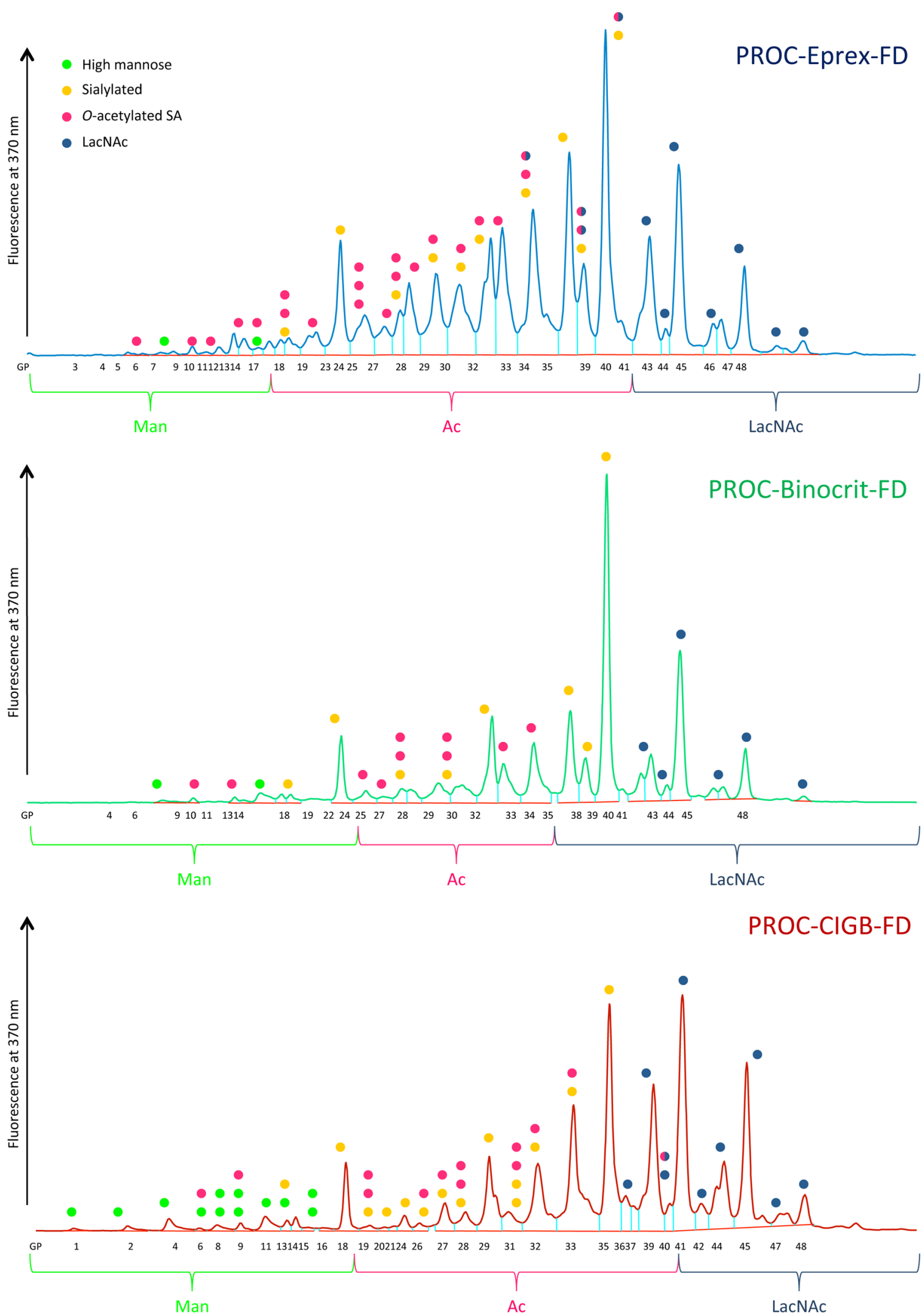

Figure 2. Fluorescence chromatogram for each PROC-labeled ESA. The brackets show where different classes of glycan are most commonly found: high mannose (green), O-acetylated (pink) and LacNAc (blue). See Figures S5-S7 for more detailed versions of these images together with Figures S9-11 that show the corresponding base peak chromatograms (BPCs) and MS/MS chromatograms.

$N$-glycan analysis (Figure 2), although it must be borne in mind that $\mathrm{SAs}$ from both $\mathrm{O}$ - and $\mathrm{N}$-glycans were released for $\mathrm{DMB}$ labeling. The presence of di-O-acetylated Neu5Ac in Eprex is spread across a range of different $\mathrm{N}$-glycan structures. Despite 

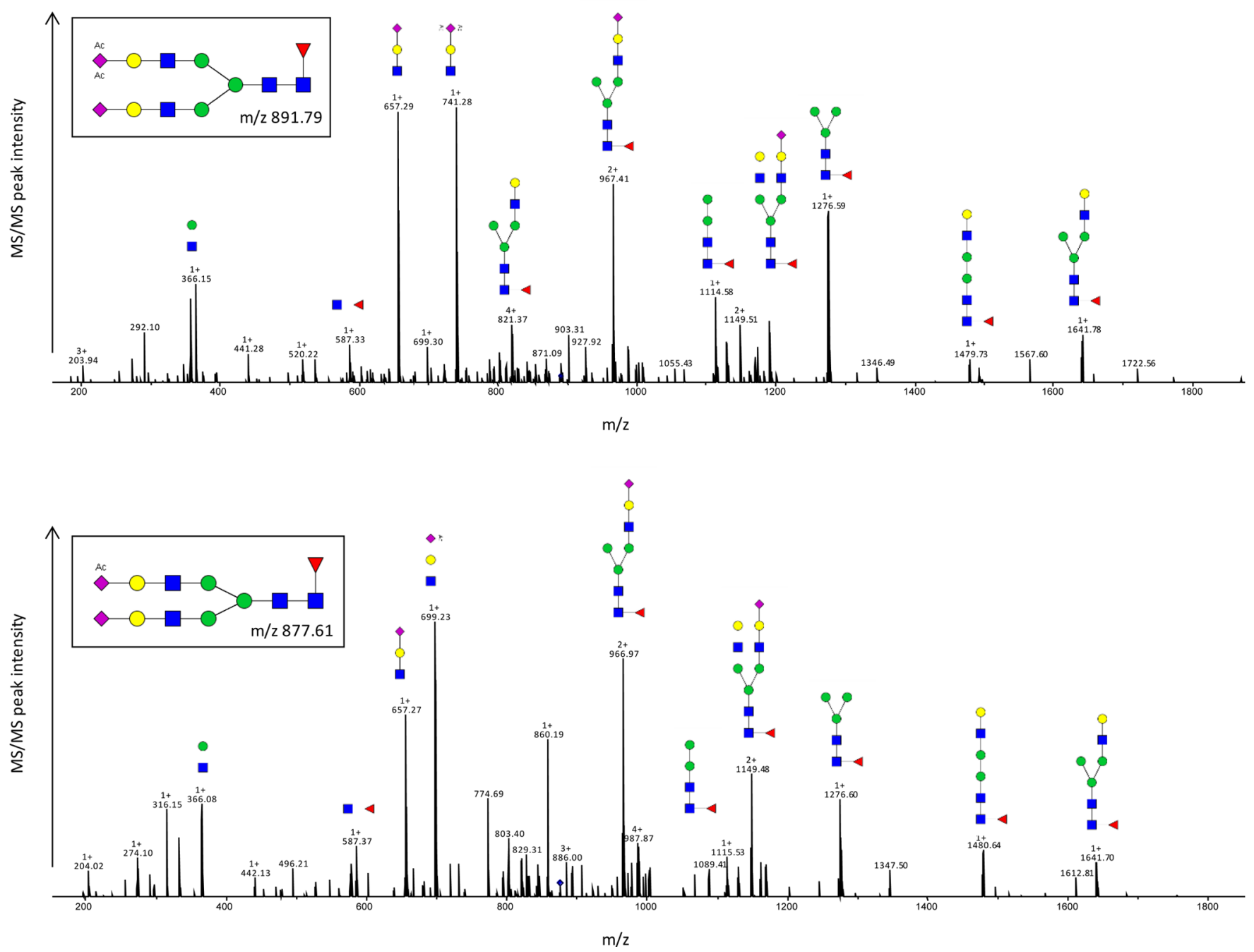

Figure 3. MS/MS spectra for $\mathrm{m} / z 891.79$ and $\mathrm{m} / z$ 877.61, showing probable structures. Symbols are used according to the Consortium for Functional Glycomics. For further details refer to Tables S10 and S11.

this, a large proportion of the $O$-acetylation in Eprex stems from the high relative abundance of $\mathrm{Neu} 5,7,9 \mathrm{Ac}_{3}(2.6 \%)$ (Figure 1). Furthermore, this high level of $O$-acetylation in Eprex is evidenced by the dramatic collapse of the complex HPLC chromatogram to a much simpler one after incubation with NanS (Figure 4). On the other hand, arguably CIGB-EPO demonstrates the greatest extent of $O$-acetylation, as it is the only one shown by PROC labeling to contain up to six $O$-acetyl groups on one glycan: FA4G4S4Ac6 (GP 11, 12, and 17 of Figure 2).

Previous work has illustrated the potential immunogenicity of Neu5Gc and the necessity to keep levels of this SA to a minimum in biotherapeutics. ${ }^{13} \mathrm{DMB}$ analysis in the current study indicates that CIGB-EPO contains the least Neu5Gc (Figure 1), which is supported by the PROC analysis (Figure S14).

The current study also confirms the presence of a highmannose-6-phosphate structure in Binocrit, previously detected by Brockmeyer et al. $^{33}$ There is limited evidence of high mannose structures in Eprex, whereas higher percentage areas of such structures are apparent in CIGB-EPO. Fucosylation of the majority of the structures in the present study has been found.
Eprex has been subjected to examination in a previous report by Shakrokh et al. ${ }^{30}$ However, the authors used a slightly different method in the form of weak anion exchange (WAX) HPLC on a $5 \mu \mathrm{m}$ column, rather than HILIC-LC analysis of PROC labeled samples. This could account for the greater level of detail found in the current study from the intact glycans and MS fragments, particularly in terms of where the $\mathrm{O}$-acetyl groups were located on the glycan, thus allowing the detection of additional structures such as FA4G4S4AcLacNAc (GP 35 of Figure 2). In addition, these earlier WAX HPLC columns yielded poorer spectral resolution. Other structural divergences, including the lack of FA2G2 and FA2G2S4LacNAc in the current analysis, could be due to batch differences. That said, initial licensing of a biosimilar is based on analysis across several batches and later batch-batch variation is strictly regulated by consistent manufacturing processes and purity and activity profiling. $^{2}$

Studies have shown that the $\mathrm{N}$-glycans as opposed to the $\mathrm{O}$ glycans are responsible for the biological activity of EPO. ${ }^{8}$ Moreover, although a number of techniques for O-glycan release are outlined in the literature, compatibility with fluorescence labeling is currently only guaranteed using hydrazinolysis. ${ }^{34}$ Thus, not only could this remove $O$-acetyl groups from SAs, but the "re- $N$-acetylation" step, for which an 

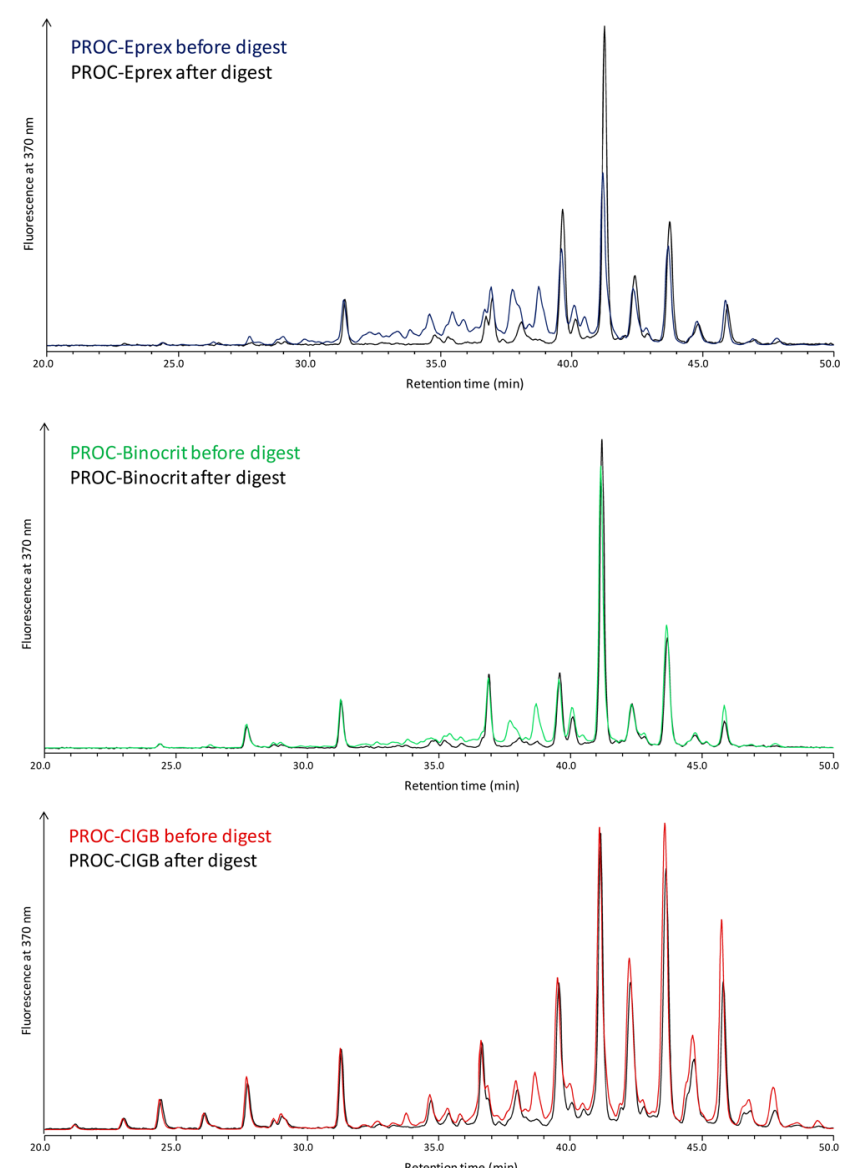

Figure 4. Fluorescence chromatograms of Eprex, Binocrit, and CIGBEPO before (blue, green and red, respectively) and after (black) incubation with an $O$-acetylesterase. The CIGB-EPO digest has previously been published by the author in collaboration with Phansopa et al. ${ }^{25}$ Part of figure adapted with permission from ref 25. Copyright 2015 The Biochemical Society.

excess of acetic anhydride is used, could acetylate hydroxyls that were underivatised in the original material. ${ }^{35}$ For these reasons, analysis of the $\mathrm{N}$-glycans was prioritised here.

It has been postulated that the hydrodynamic size of EPO influences its in vivo bioactivity. ${ }^{36}$ This not only depends on the SA content but also the LacNAc composition that forms either branched or repeat units. To the authors' knowledge, to date there is no literature outlining the effect on bioavailability of the presence of branching compared to extensions of LacNAc. However, it is widely accepted that branching leads to improved in vivo bioactivity, but longer chains result in quicker clearance. $^{10,37}$ Glycoproteins containing LacNAc repeats with terminal sialylation or fucosylation are ligands for selectins, which could therefore play a role in EPO behavior. ${ }^{38}$ Although in this study it cannot be definitively stated that, for example, structure FA4G4S3LacNAc is not in fact FA3G3S3(LacNac)2 (GP 38-40 of Figure 2), it is still worth noting that a similar pattern of LacNAc repeats/branching is seen between the ESA products. Bisecting glycans were not expected using this expression system.

Particular aspects of the $\mathrm{N}$-glycans of ESAs can be fine-tuned by the alteration of cell line production conditions, for example, the SA content: $\mathrm{CHO}$ cells can be engineered to express either more sialidase or more sialyltransferase; thus, the glycans are sialylated to a lesser or greater extent, respectively. ${ }^{40}$ Changes to cell growth conditions in the bioreactor could also change glycosylation features. Studies such as the present one will help to build a picture of the most desirable GCQAs that an ESA product should have, thereby increasing the efficacy while maintaining quality and safety.

Aside from $O$-acetylesterase, complementary exoglycosidase digestions could yield more detail and confirm assignments made by LC-MS analysis. Fucosidases could be used to check for outer-arm fucose moieties and galactosidases and sialidases of different specificity would help to determine the linkage patterns between substituents.

In the present study, solely alpha epoetins were analyzed, but in a further study, it could be beneficial to see whether other ESA biosimilars for example NeoRecormon (beta), Eporatio (theta), or Retacrit (zeta), offer similar $O$-acetylation patterns. Indeed, such an analysis could be extended to other biotherapeutics; one possibility is follicle stimulating hormone (FSH) or the recently licensed monoclonal antibody biosimilar Infliximab. Some differences between the Eprex of the present study and the Eprex of Shahrokh et al. were noted, which could be due to batch differences and therefore a study across different batches of the same ESA could give further insight.

\section{CONCLUSIONS}

Three ESA products, namely, Eprex, Binocrit, and CIGB-EPO, have been subjected to three forms of analysis to compare GCQAs. First, through RP-HPLC analysis of acid-released, DMB labeled SA derivatives, it has been demonstrated that Eprex contains the greatest relative abundance of $O$-acetylated derivatives, mainly, because of high levels of Neu5,9 $\mathrm{Ac}_{2}$ and $\mathrm{Neu} 5,7,9 \mathrm{Ac}_{3}$. Binocrit has been found to contain the least Neu5Gc, a SA known to evoke an immune response in humans. Second, PROC labeled $N$-glycans run on HILIC-UHPLC-MS also showed high levels of $O$-acetylation, with up to six $O$-acetyl groups on one structure seen in CIGB-EPO. Finally, peak assignment was confirmed by $\mathrm{O}$-acetylesterase digestion. This $\mathrm{O}$-acetylation, alongside fucosylation and LacNAc repeats, could influence lifetime in the bloodstream (as hepatic removal could be regulated through the presence of $O$-acetyl groups on the SA), as well as affect the quality and safety of the ESA products.

Outlined in this Article for the first time is a detailed analysis of PROC-labeled glycans from ESAs, achieved with automated sample preparation. Automated sample preparation was also used for the first time for DMB analysis and the relative abundance of each SA derivative was calculated. The employed combination of methods made the recognition of distinct glycan features possible, such as a SA with one or two $\mathrm{O}$-acetyl groups in the presence of a SA with no extra acetylation.

In the case of fully licensed Eprex and Binocrit, the above characteristics may only now be being observed but should not be a cause for concern unless definitively linked to adverse effects. Binocrit has previously been shown to contain highmannose-phosphate structures; therefore, their presence in CIGB-EPO may not be a disadvantage but must be coupled with further analysis and clinical trial to conclusively determine its effect. Taking into account the differences between ostensibly similar products and the relative ease of analysis afforded by the methods used in the current study, it is recommended that all ESAs be analyzed to this level of detail in the future. 


\section{ASSOCIATED CONTENT}

\section{S Supporting Information}

The Supporting Information is available free of charge on the ACS Publications website at DOI: 10.1021/acs.analchem.7b00353.

Detailed methods for SA and $N$-glycan release and labeling, DMB labeling repeatability data, overlays of HPLC chromatograms of DMB-labeled SA (3 runs) and PROC-labeled N-glycans (4 runs) for each ESA, larger, more detailed versions of the images in Figure 2, together with the corresponding MS/MS chromatograms, calculation of RSD for DMB- and PROC-labeled samples, as well as tables presenting PROC-glycan MS fragment identification (PDF)

\section{AUTHOR INFORMATION}

\section{Corresponding Authors}

*Phone: +44 (0) 1865408 554. Fax: +44 (0) 870163 4620. Email: Daniel.Spencer@ludger.com.

*Phone: +44 (0) 118378 7338. E-mail: h.m.i.osborn@reading. ac.uk.

\section{ORCID}

Helen M. I. Osborn: 0000-0002-0683-0457

\section{Author Contributions}

All authors have given approval to the final version of the manuscript.

\section{Funding}

This work was funded by the BBSRC Case Award BB/ K012673/1 and Ludger Ltd. The robotics work was partially funded by the European Union's Seventh Framework Program (FP7-Health-F5-2011) under Grant agreement 278535 (HighGlycan).

\section{Notes}

The authors declare the following competing financial interest(s): D.L.F. is the chief executive of Ludger, Ltd. D.I.R.S. and R.A.G. work for Ludger, Ltd., a commercial glycobiology company. The remaining authors declare no competing financial interest.

\section{ACKNOWLEDGMENTS}

With thanks to Antonio Vallin, Center for Molecular Immunology, Cuba for provision of the CIGB-EPO.

\section{REFERENCES}

(1) Naughton, B. A.; Kaplan, S. M.; Roy, M.; Burdowski, A. J.; Gordon, A.; Piliero, S. J. Science 1977, 196 (4287), 301-302.

(2) Schellekens, H. NDT Plus 2009, 2 (Suppl 1), i27-i36.

(3) Reichel, C. Drug Test. Anal. 2013, 5, 877-889.

(4) Lamon, S.; Giraud, S.; Egli, L.; Smolander, J.; Jarsch, M.; Stubenrauch, K.-G.; Hellwig, A.; Saugy, M.; Robinson, N. J. Pharm. Biomed. Anal. 2009, 50 (5), 954-958.

(5) Tsiftsoglou, A. S.; Ruiz, S.; Schneider, C. BioDrugs 2013, 27 (3), 203-211.

(6) Egrie, J. C.; Browne, J. K. Br. J. Cancer 2001, 84 (Suppl 1), 3-10.

(7) Skibeli, V.; Nissen-Lie, G.; Torjesen, P. Blood 2001, 98 (13), 3626-3634.

(8) Higuchi, M.; Oh-eda, M.; Kuboniwa, H.; Tomonoh, K.; Shimonaka, Y.; Ochi, N. J. Biol. Chem. 1992, 267 (11), 7703-7709.

(9) Wasley, L. C.; Timony, G.; Murtha, P.; Stoudemire, J.; Dorner, A. J.; Caro, J.; Krieger, M.; Kaufman, R. J. Blood 1991, 77 (12), 26242632.
(10) Yanagihara, S.; Taniguchi, Y.; Hosono, M.; Yoshioka, E.; Ishikawa, R.; Shimada, Y.; Kadoya, T.; Kutsukake, K. Biol. Pharm. Bull. 2010, 33 (9), 1596-1599.

(11) Corfield, A. P.; Sander-Wewer, M.; Veh, R. W.; Wember, M.; Schauer, R. Biol. Chem. Hoppe-Seyler 1986, 367, 433-439.

(12) Llop, E.; Gutiérrez-Gallego, R.; Segura, J.; Mallorquí, J.; Pascual, J. a. Anal. Biochem. 2008, 383 (2), 243-254.

(13) Ghaderi, D.; Taylor, R.; Padler-Karavani, V.; Diaz, S.; Varki, A. Nat. Biotechnol. 2010, 28 (8), 863-867.

(14) EMEA. Binocrit EMEA scientific discussion, 2007. http://www. ema.europa.eu/docs/en_GB/document_library/EPAR_-_Scientific Discussion/human/000725/WC500053615.pdf.

(15) Pérez-Oliva, J. F.; Casanova-González, M.; García-García, I.; Porrero-Martín, P. J.; Valenzuela-Silva, C. M.; Hernández-Montero, T.; Lagarde-Ampudia, M.; Casanova-Kutsareva, Y.; Avila-Albuerne, Y.; Vargas-Batista, A.; Bobillo-López, H.; Herrera-Valdés, R.; López-Saura, P. A. BMC Nephrol. 2005, 6, 5.

(16) Brinks, V.; Hawe, A.; Basmeleh, A. H. H.; Joachin-Rodriguez, L.; Haselberg, R.; Somsen, G. W.; Jiskoot, W.; Schellekens, H. Pharm. Res. 2011, 28 (2), 386-393.

(17) World Health Organization; WHO Document Production Services: Geneva, 2013; pp 1-65.

(18) Berghout, A. Biologicals 2011, 39 (5), 293-296.

(19) Harvey, D. J.; Royle, L.; Radcliffe, C. M.; Rudd, P. M.; Dwek, R. A. Anal. Biochem. 2008, 376 (1), 44-60.

(20) Hara, S.; Takemori, Y.; Yamaguchi, M.; Nakamura, M.; Ohkura, Y. Anal. Biochem. 1987, 164 (1), 138-145.

(21) Ventham, N. T.; Gardner, R. A.; Kennedy, N. A.; Shubhakar, A.; Kalla, R.; Nimmo, E. R.; Fernandes, D. L.; Satsangi, J.; Spencer, D. I. R. PLoS One 2015, 10 (4), e0123028.

(22) Royle, L.; Radcliffe, C. M.; Dwek, R. a; Rudd, P. M. Methods Mol. Biol. 2006, 347, 125-143.

(23) Kozak, R. P.; Tortosa, C. B.; Fernandes, D. L.; Spencer, D. I. R. Anal. Biochem. 2015, 486, 38-40.

(24) Guile, G. R.; Rudd, P. M.; Wing, D. R.; Prime, S. B.; Dwek, R. A. Anal. Biochem. 1996, 240 (2), 210-226.

(25) Phansopa, C.; Kozak, R. P.; Liew, L. P.; Frey, A. M.; Farmilo, T.; Parker, J. L.; Kelly, D. J.; Emery, R. J.; Thomson, R. I.; Royle, L.; Gardner, R. A.; Spencer, D. I. R.; Stafford, G. P. Biochem. J. 2015, 472 (2), 157-167.

(26) Royle, L.; Mattu, T. S.; Hart, E.; Langridge, J. I.; Merry, A. H.; Murphy, N.; Harvey, D. J.; Dwek, R. A.; Rudd, P. M. Anal. Biochem. 2002, 304 (1), 70-90.

(27) Klein, A.; Diaz, S.; Ferreira, I.; Lamblin, G.; Roussel, P.; Manzi, a E. Glycobiology 1997, 7 (3), 421-432.

(28) Royle, L.; Campbell, M. P.; Radcliffe, C. M.; White, D. M.; Harvey, D. J.; Abrahams, J. L.; Kim, Y. G.; Henry, G. W.; Shadick, N. A.; Weinblatt, M. E.; Lee, D. M.; Rudd, P. M.; Dwek, R. A. Anal. Biochem. 2008, 376 (1), 1-12.

(29) Watson, E.; Bhide, A.; van Halbeek, H. Glycobiology 1994, 4 (2), 227-237.

(30) Shahrokh, Z.; Royle, L.; Saldova, R.; et al. Mol. Pharmaceutics 2011, 8, 286-296.

(31) Vandamme-Feldhaus, V.; Schauer, R. J. Biochem. 1998, 124 (1), 111-121.

(32) Ashwell, G.; Morell, A. G. Adv. Enzymol. Relat. Areas Mol. Biol. 2006, 41, 99-127.

(33) Brockmeyer, C.; Seidle, A. Eur. J. Hosp. Pharm. Pract. 2009, 15 (2), 34-40.

(34) Zauner, G.; Kozak, R. P.; Gardner, R. A.; Fernandes, D. L.; Deelder, A. M.; Wuhrer, M. Biol. Chem. 2012, 393 (8), 687-708.

(35) Merry, A. H.; Neville, D. C. A.; Royle, L.; Matthews, B.; Harvey, D. J.; Dwek, R. A.; Rudd, P. M. Anal. Biochem. 2002, 304 (1), 91-99.

(36) Elliott, S.; Egrie, J.; Browne, J.; Lorenzini, T.; Busse, L.; Rogers, N.; Ponting, I. Exp. Hematol. 2004, 32 (12), 1146-1155.

(37) Takeuchi, M.; Inoue, N.; Strickland, T. W.; Kubota, M.; Wada, M.; Shimizu, R.; Hoshi, S.; Kozutsumi, H.; Takasaki, S.; Kobata, a. Proc. Natl. Acad. Sci. U. S. A. 1989, 86 (20), 7819-7822.

(38) Vestweber, D.; Blanks, J. E. Physiol. Rev. 1999, 79 (1), 181-213. 
(39) Grabenhorst, E.; Schlenke, P.; Pohl, S.; Nimtz, M.; Conradt, H. S. Glycoconj. J. 1999, 16 (2), 81-98.

(40) Butler, M.; Spearman, M. Curr. Opin. Biotechnol. 2014, 30, 107112. 